\title{
Mixed reality-based simulator for training on imageless navigation skills in total hip replacement procedures.
}

\author{
Mara Catalina Aguilera-Canon ${ }^{1}$, Tom Wainwright ${ }^{2}$, \\ Xiaosong Yang ${ }^{1}$, Hammadi Nait-Charif ${ }^{1}$, \\ ${ }^{1}$ Faculty of Media and Communications, \\ ${ }^{2}$ Faculty of Health and Social Sciences \\ Bournemouth University, Bournemouth , United Kingdom \\ \{maguileracanon, twainwright, xyang, hncharif\}@bournemouth.ac.uk
}

\begin{abstract}
.
Imageless navigation systems (INS) in orthopaedics have been used to improve the outcomes of several orthopaedic procedures such as total hip replacement [1] [2]. However, the increased surgical times and the associate learning curve discourage surgeons from using navigation systems in their theatres [2]. This paper presents a Mixed Reality (MR) simulator that helps surgeons acquire the infrared based navigation skills before performing it in reality.. A group of 7 hip surgeons tried the application, expressing their satisfaction with all the features and confirmed that the simulator represents a cheaper and faster option to train surgeons in the use of INS than the current learning methods.
\end{abstract}

Keywords: Imageless navigation, holographic, Augmented Reality, Mixed Reality, machine vision, surgical training.

\section{Introduction}

Total hip replacement is one of the most successful and cost-effective surgeries in the orthopaedic field with over 66,000 procedures performed each year in England [3]. Imageless navigation systems (INS) provide an alternative to conventional methods in achieving a more accurate position of the implants [4][5] minimizing the amount of radiation the patient is exposed to, as well as the risks of leg length discrepancies, dislocation, a higher wear rate and other clinical complications[6].

In order to give the system the right spatial data input and avoid further orientation mistakes intraoperatively, surgeons and trainees must learn to master the skills needed for a proper use of the infrared tracking camera. Unfortunately while surgeons learn to adapt their instrument movements to be recognized by the infrared cameras, surgical times can be extended[5][7], however this amount of extra can be shorten up to $4.8+/-3.8$ minutes after significant amount of practice [2].

Mixed and Augmented reality has been applied into the medical training field due to their standalone nature and spatial understanding capabilities. Among some examples are the company CAE Healthcare[8] and Fundmaental VR [9], which have developed holographic interfaces to train medial staff in the use of diagnostic ultrasound, anatomy and surgical approaches. However, to the date and to the best of our knowledge there is no existing MR simulator to help surgeons train in the use of 
navigation systems without requiring the navigation system itself or cadavers. This paper presents a mixed reality-based simulator, which allows orthopaedic surgeons to practice tool's manipulation skills required in INS based surgery before practicing in real theatres.

\section{Materials and methods}

\subsection{HoloLens mixed reality and PTC Vuforia ${ }^{\text {TM }}$ Engine}

Our holographic application was designed to train surgeons on the basic skills needed during the calibration stage of INS, namely the awareness of the infrared camera's tracking volume and how to manipulate surgical instruments in hardly to reach places while avoiding targets occlusion. We chose the HoloLens due to its head 6-DoF tracking capabilities and ability to render the digital content according to the spatial understanding information. To optimize development times some of the Mixed Reality toolkit [10] shader libraries and input functions were included during the building stage of the application.

The Vuforia Engine implements machine vision algorithms for real-time tracking of images with certain characteristics folded around different volumes. The desired image-based markers must be pre-processed using the Vuforia Target Manager web tool to extract "sharp, pointed, chiselled detail in the image" [11] called features which will be used to determine the location and orientation of a given target in real time. Two raw images were used as different types of markers and incorporated into the simulator. For both targets high density and uniformly distributed features were used while avoiding repetitive patterns. A plain image target was chosen to help the user locate the surgical scenario on the desired real space coordinates while an image folded as a cylindrical target was chosen for the pointer since its shape provides the camera a continuous projected area at different rotations around its own axis.

\subsection{Application design}

During real procedures, the infrared camera (IR) cast rays which are reflected at the surface of some passive spheres attached in a specific position on the surface of a unique mount. To make objects always visible to the camera, this mount has to be pointing in a similar direction as the camera view and inside its tracking volume. In our model each spheres' mount (Fig. 1$)$ has a vector normal to its surface $\left(\widehat{N}_{\text {tool }}\right.$ and $\left.\widehat{N}_{\text {frame }}\right)$ and the direction of the camera view is also represented by the vector

$\widehat{N}_{\text {camera view }}$. Once the users anchor the holographic environment in their real-world they ensure that their workplace is inside the camera field of view by modifying the orientation of the camera 3D model thanks to the device's gesture recognition API.

The acquisition of the points or anatomical landmarks on the surface of the pelvis and femur bone are the most important steps during the calibration of INS, since it allows the system to define the anatomical planes in which the angles of resurfacing and acetabular cup implant insertion will be projected. At this stage an important skill 
to transfer is to be aware of holding the surgical instruments inside the (IR) camera tracking volume and on its field of view. Therefore, in our simulation, the system will be able to register points, only when the angle between the normal vectors of the fixed frame and pointer tool and the camera view are less or equal to $45^{\circ}$.

Finally, the app provides the users an interface of the tools orientation measured on the two anatomic planes calculated from their landmark registration, namely the coronal $^{1}$ and sagittal ${ }^{2}$ plane. Additionally, a dynamically coloured line is casted from the tip of the reaming tool in the direction $D_{\text {tool }}$, changing its colour according to how close the trainee is to an orientation inside a "safe zone" [4].

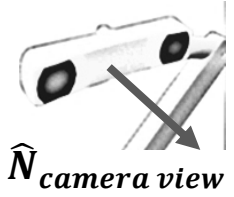

(a)

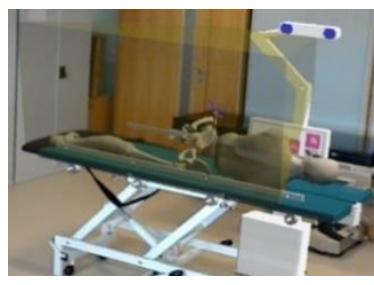

(d)

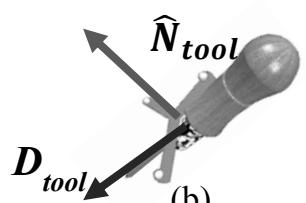

(b)

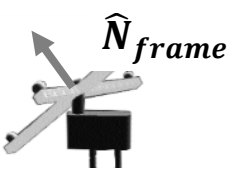

(c)

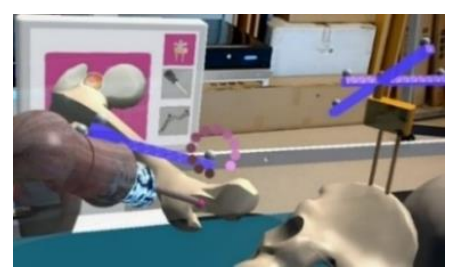

(e)

Fig. 1. (a) $3 \mathrm{D}$ model of the infrared camera, $\tilde{N}_{\text {camera view }}$ is the vector normal to the frontal face of the camera. (b) 3D model attached to the cylindrical tracked object, $\tilde{N}_{\text {tool }}$ is the normal of the mount surface and $D_{\text {tool }}$ a vector in direction of the cylinder's axis. (c) Frame of reference with a vector normal to the surface on which the reflective spheres are supported.(d) Overall view of surgical scene (e) Point registration stage

\section{Evaluation}

A group of 7 hip surgeons and surgical trainees with previous knowledge about INS were invited to try the simulator. During the study they were asked to set up the surgical scene, adjust the infrared camera field of view and acquire a total of 27 points which were displayed one after another and distributed between pelvic anatomical landmarks, the surface of the acetabulum and femoral landmarks. These high amounts of points enabled learning through repetition, since the simulator would only allow the user to progress in the experience as a new point was acquired, visual and audio feedback were displayed after a successful landmark acquisition, allowing users to

\footnotetext{
${ }^{1}$ Plane that divides the body into front and back sections.

${ }^{2}$ Plane that divides the body into left and right sections.
} 
quickly understand that the absence of registration success was translated as a wrong instrument manipulation leading them to modify their instrument's pose quickly.

\subsection{Results and discussion}

In our study all participants went successfully through all the simulation stages without needing extra help. In addition, all of them agreed that the simulator allows an understanding of the skills needed in INS, furthermore 5 out of the 7 participants believed that the application was easy to use. Moreover, all of them considered that the visual content was attractive and three of them strongly agreed with this affirmation. Finally, for 5 out of the 7 participants, the cylindrical marker was tracked with minimal loss of tracking and one of them reported no loss of tracking at all during the entire experience.

The simulator represents a valuable practice for both surgeons with some or none previous knowledge about intraoperative navigation systems. We have used game technologies to create a novel proposal of a functional MR based simulator to help trainee surgeons to understand the principles of imageless navigation systems without high-budget investments

\section{References}

1. Snijders, T., Gaalen, S., Gast, A.: Precision and accuracy of imageless navigation versus freehand implantation of total hip arthroplasty: A systematic review and meta-analysis. The International Journal of Medical Robotics and Computer Assisted Surgery. (2017).

2. Thorey, F., Klages, P., Lerch, M., Flörkemeier, T., Windhagen, H., von Lewinski, G.: Cup positioning in primary total hip arthroplasty using an imageless navigation device: is there a learning curve? Orthopedics. 32, 14-7 (2009).

3. Registry, N.J: NJR StatsOnline, http://www.njrcentre.org.uk/njrcentre/Healthcareproviders/Accessingthedata/StatsOnline/ NJRStatsOnline/tabid/179/Default.aspx.

4. Chang, J.-D., Kim, I.-S., Bhardwaj, A.M., Badami, R.N.: The Evolution of ComputerAssisted Total Hip Arthroplasty and Relevant Applications. Hip $1 \&$ Pelvis. 29, 1-14 (2017).

5. Schnurr, C., Michael, J.W.P., Eysel, P., König, D.P.: Imageless navigation of hip resurfacing arthroplasty increases the implant accuracy. Int Orthop. 33, 365-72 (2009).

6. Paprosky, W.G., Muir, J.M.: Intellijoint HIP: a 3D mini-optical navigation tool for improving intraoperative accuracy during total hip arthroplasty. Medical devices (Auckland, NZ). 9, 401 (2016).

7. Silvennoinen, M., Helfenstein, S., Ruoranen, M., Saariluoma, P.: Learning basic surgical skills through simulator training. Instructional Science. 40, 769-783 (2012).

8. Healthcare, C.: VimedixAR, https://caehealthcare.com/hololens.

9. VR, F.: Multiperson Medical Training, ttps://www.fundamentalvr.com/services/hololensstudio/.

10. Microsoft-OpenSource: MixedRealityToolkit-Unity, https:/github.com/Microsoft/MixedRealityToolkit-Unity.

11. Vuforia, P.: Vuforia Developer Library, https://library.vuforia.com/articles/Training/Image-Target-Guide.html. 